Original Article

\title{
Protective Mechanisms of Suxiao Jiuxin Pills (速效救心丸) on Myocardial Ischemia-Reperfusion Injury in vivo and in vitro*
}

\author{
TAN Ya-fang ${ }^{1,2}$, YU Juan ${ }^{3}$, PAN Wen-jun ${ }^{1,2}$, QI Jian-yong ${ }^{1,2}$, and ZHANG Min-zhou ${ }^{1,2}$
}

\begin{abstract}
Objective: To study the protective mechanism of Chinese medicine Suxiao Jiuxin Pills (速效救 心丸, SXJ) on myocardial ischemia and reperfusion (I/R) injury. Methods: Mouse myocardial I/R injury model was created by 30 -min coronary artery occlusion followed by $24-\mathrm{h}$ reperfusion, the mice were then divided into the sham group ( $n=7$ ), the I/R group ( $n=13$ ), the tirofiban group (TIR, positive drug treatment, $n=9$ ), and the SXJ group ( $n=11)$. Infarct size (IS), risk region (RR), and left ventricle (LV) were analyzed with double staining methods. In addition, $\mathrm{H} 9 \mathrm{C} 2$ rat cardiomyocytes were cultured with $\mathrm{Na}_{2} \mathrm{~S}_{2} \mathrm{O}_{4}$ to simulate $\mathrm{I} / \mathrm{R}$ in vitro. The phosphorylation of extracellular regulated protein kinases $1 / 2$ (ERK1/2), protein kinase $B(A K T)$, glycogen synthase kinase-3 $\beta$ (GSK3 $\beta$ ), and protein expression of GATA4 in nucleus were detected with Western blot assay. Results: The ratio of IS/RR in SXJ and TIR groups were lower than that in I/R group (SXJ, 22.4\% $\pm 6.6 \%$; TIR, 20.8\% $\pm 3.3 \%$; vs. I/R, 35.4\% $\pm 3.7 \%, P<0.05$, respectively). In vitro experiments showed that SXJ increased the $\mathrm{Na}_{2} \mathrm{~S}_{2} \mathrm{O}_{4}$-enhanced phosphorylation of AKT/GSK3 $\beta$ and nuclear expression of GATA4. Conclusion: SXJ prevents myocardial I/R injury in mice by activating AKT/GSK3 $\beta$ and GATA4 signaling pathways.

KEYWORDS myocardial ischemia and reperfusion injury, Suxiao Jiuxin Pills, GATA4, Chinese medicine, mouse
\end{abstract}

Percutaneous coronary intervention $(\mathrm{PCl})$ is a milestone in treating acute myocardial infarction (AMI). Early reperfusion can instantaneously open the occluded coronary artery, rescuing the ischemic myocardium. ${ }^{(1)}$ However, myocardial ischemia and reperfusion (I/R) injury, which can trigger death of myocytes, seriously affects clinical effectiveness of $\mathrm{PCl}$. An animal study showed that $50 \%$ of the infarct area were caused by myocardial I/R injury. ${ }^{(2)}$ Many signaling molecules and mechanisms are postulated to modulate myocardial I/R injury, such as calcium overload, reactive oxygen species, energy metabolism dysfunction of myocardial fibers, cellular adhesion molecules, neutrophils, vascular endothelial cells, and apoptosis, etc. ${ }^{(1)}$ Several agents, such as freeradical scavengers and inhibitors of the immune system, have been shown to protect heart from $\mathrm{I} / \mathrm{R}$ injury. Although it has been actively studied for more than 35 years, myocardial I/R injury is still not yet treated effectively. The high mortality and non-availability of suitable drug treatments led to heightened interest in developing early stage therapies to prevent death of myocytes following myocardial $I / R$, and to improve the life span and quality in patients after $\mathrm{AMI}^{\left({ }^{(3)}\right.}$

Suxiao Jiuxin Pill (速效救心丸, SXJ), which formula can be found in the latest 2015 Chinese Pharmacopoeia, is made up of 2 herbs, Rhizoma Chuanxiong and Borneolum. ${ }^{(4)}$ SXJ contains 4 active ingredients: ferulic acid, menthol, borenol, and isoborneol; and is widely used for treating AMI and angina pectoris in China for more than 30 years. ${ }^{(5)}$ Previous publications reported that SXJ could alleviate acute myocardial ischemia, prevent the formation of atherosclerosis (AS) and induce potent relaxation and inhibition on contraction in human artery. ${ }^{(6-8)}$

(C)The Chinese Journal of Integrated Traditional and Western Medicine Press and Springer-Verlag GmbH Germany, part of Springer Nature 2020

*Supported by the National Natural Science Foundation of China (No. 81473471 and No. 81603429) and Foundation of Guangdong Hospital of Chinese Medicine (No. YK2013B2N11, No. YN2014ZH01, No. YN2014ZHR203, and No. YN2016QJ19). 1. AMI Key Laboratory of Chinese Medicine in Guangzhou, Guangdong Province Hospital of Chinese Medicine, the 2nd Affiliated Hospital of Guangzhou University of Chinese Medicine, Guangdong Provincial Academy of Chinese Medical Science, Guangzhou (510006), China; 2. Intensive Care Research Team of Traditional Chinese Medicine, Guangdong Province Hospital of Chinese Medicine, the 2nd Affiliated Hospital of Guangzhou University of Chinese Medicine, Guangdong Provincial Academy of Chinese Medical Science, Guangzhou (510006), China; 3. Animal Laboratory, Guangdong Province Hospital of Chinese Medicine, Guangzhou (510006), China

Correspondence to: Prof. ZHANG Min-zhou, E-mail: minzhouzhang8@163.com

DOI: https://doi.org/10.1007/s11655-020-2726-2 
Despite its extensive clinical usages, seldom report is available for the detailed effects and underlying mechanisms of SXJ on myocardial I/R injury. In the present study, we explored roles of SXJ on myocardial I/R injury by studying its effects on infarct size (IS), risk region (RR), and left ventricle (LV) in experimental $\mathrm{I} / \mathrm{R}$ mice and rats, and by evaluating its effects on the classic reperfusion injury salvage kinase (RISK) I/R injury signaling pathway in vivo and in vitro.

\section{METHODS}

\section{Animals and Reagents}

This study was performed in accordance with the guidelines and with approval from the Institutional Animal Care and Use Committee of Guangdong Province Hospital of Chinese Medicine, Guangzhou University of Traditional Chinese Medicine, and with the Guide for the Care and Use of Laboratory Animals (Department of Health and Human Services, National Institutes of Health, Publication No. 86-23, revised 1996).

A total of 120 male wild-type C57BL/6J mice (10 to 12 weeks old, $25 \pm 5 \mathrm{~g}$ body weight) and 60 male wild-type SD rats (10 to 12 weeks old, $250 \pm 20 \mathrm{~g}$ body weight) were obtained from the Experimental Animal Center of Guangdong Province. Triphenyltetrazolium chloride (TTC) and Evans blue were purchased from DingGuo Biotechnology Corp (Beijing, China); 10\% neutral buffered formalin were purchased from WEX Corp (Guangzhou, China); pentobarbital sodium were purchased from Sigma-Aldrich Corp (Guangzhou, China).

\section{Preparation of SXJ}

SXJ was provided by the Sixth Chinese Drugs Factory of Tianjin Zhongxin Pharmaceutical Co., Ltd. (Tianjing, China, batch No. 6015152). Main active components of SXJ are ferulic acid $\left(\mathrm{C}_{10} \mathrm{H}_{10} \mathrm{O}_{4}\right)$, menthol $\left(\mathrm{C}_{10} \mathrm{H}_{20} \mathrm{O}\right)$, borenol $\left(\mathrm{C}_{10} \mathrm{H}_{18} \mathrm{O}\right)$, and isoborneol $\left(\mathrm{C}_{10} \mathrm{H}_{18} \mathrm{O}\right)$. Each pill of SXJ weighs $40 \mathrm{mg}$, contains $3.5 \mathrm{mg}$ borneol and $24.9 \mu \mathrm{g}$ ferulic acid, as determined with following methods: (1) The content of ferulic acid was determined by high performance liquid chromatography (HPLC) using Agilent 1200. Chromatographic column was a C18 column (4.6 mm $\times 250 \mathrm{~mm}, 2.6 \mu \mathrm{m}, \mathrm{YMC}-\mathrm{C} 18$ ). The mobile phase was composed of methanol, water and acetic acid (30:70:1). The ultraviolet (UV) detection wavelength was $321 \mathrm{~nm}$. The number of theoretical plates should be calculated at no less than 5000 according to the ferulic acid peak. Reference and test solutions
(10 $\mu \mathrm{L}$ each) were injected to liquid chromatography separately. (2) The content of borneol was determined by gas chromatography method: The gas chromatography was conducted on Agilent 7890A. A polyethylene glycol 20000 (PEG-20M) capillary column $(0.53 \mathrm{~mm} \times 30 \mathrm{~mm}$, $1.0 \mu \mathrm{m}$, DB-WAX) was used for the separation. The chromatographic conditions were as follows: the column temperature is $150{ }^{\circ} \mathrm{C}$, the inlet temperature is $200{ }^{\circ} \mathrm{C}$, and the detector temperature is $200{ }^{\circ} \mathrm{C}$. The number of theoretical plates should be calculated to be more than 5000 according to the peak value of borneol. Menthol was used as internal standard. The injection volume was $1 \mu \mathrm{L}$.

\section{Myocardial I/R Model and Phase I Protocol in Mice}

The murine model of $\mathrm{I} / \mathrm{R}$ has been described in detail in our previous studies. ${ }^{(9-11)}$ Briefly, mice were anesthetized with sodium pentobarbital $(60 \mathrm{mg} / \mathrm{kg}$, intraperitoneal injection, i.p), intubated, and ventilated with room air at a rate of 110 strokes/min and with a tidal volume of $0.25 \mathrm{~mL}$ using a mouse ventilator (Inspira, Harvard Apparatus, Holliston, MS, USA). The chest was opened through a left thoracotomy under a dissecting microscope. An 8-0 nylon suture was passed under the mid-left anterior descending coronary artery (LAD, 2-3 mm inferior to the left auricle) and a nontraumatic occluder was applied on the artery. Ischemia was elicited by a 30-min coronary occlusion followed by 24-h reperfusion. Significant changes, including widening of the QRS complex and elevation of ST segment in electrocardiography were indicators of successful coronary occlusion. The chest was closed in layers, and animals were weaned from the ventilator when they resumed spontaneous breathing.

In the phase I protocol (Figure 1A), mice were assigned to 4 groups: the sham group $(n=7)$, I/R group ( $n=13)$, the tirofiban group (TIR, $n=9$ ), and the SXJ group $(n=11)$. Mice in the sham group received saline intraperitoneal injection and all surgery procedures except LAD occlusion; mice in I/R group were subjected to saline intragastric administration and I/R surgery; TIR mice received tirofiban $5 \mathrm{mg} / \mathrm{kg}$, intraperitoneal injection, as a positive control of the protective effects for drug-induced ischemia preconditioning. Based on literature, clinical usage, and the Meeh-Rubner equation of dose conversion between humans and mice, SXJ group received $750 \mathrm{mg} / \mathrm{kg}$ SXJ intragastric administration daily for 3 days following $\mathrm{I} / \mathrm{R}$ surgery. 


\section{Myocardial Ischemia Injury Model in Rats}

To further confirm the effects of SXJ on myocardial injury in vivo, a rat model by isoproterenol (ISO, $85 \mathrm{mg} / \mathrm{kg}$ for 2 days) subcutaneous administration was created. Serum levels of cardiac troponin I (cTnl), creatine kinase (CK), CK-MB, lactate dehydrogenase (LDH), aspartate transaminase (AST), and $\alpha$-hydroxybutyric dehydrogenase ( $\alpha-\mathrm{HBD})$. Rats in the control group received saline intraperitoneal injection (i.p) and all the procedures except ISO administration; rats in ISO group were subjected to ISO administration (i.p) for 2 days; MET rats received metoprolol, $10 \mathrm{mg} / \mathrm{kg}$ per day i.p, for 3 days prior to and 2 days after ISO stimulation. SXJ group received $750 \mathrm{mg} / \mathrm{kg} \mathrm{SXJ}$ intragastric administration daily for 3 days following ISO stimulation (totally 5 days).

\section{Heart Rhythm Analysis}

Continuous electrocardiographic monitoring (RM6240; ChengDu Instruments) was performed during in vivo myocardial I/R with LAD ligation. Heart rate and rhythm were analyzed throughout the experiment. The incidence and type of arrhythmias, including atrial premature beats, heart block, ventricular premature contractions and ventricular tachycardia, were evaluated during I/R based on limb lead recordings.

\section{In vitro Tissue Staining and Infarct Size Measurement}

At the end of 24-h reperfusion, the heart was perfused with $1 \times$ phosphate buffer solution $(1 \times \mathrm{PBS}$, $\mathrm{pH}$ 7.4) through an aortic cannula. The ligature around the LAD was retied. Evans blue dye was diluted to $1 \%(\mathrm{v} / \mathrm{v})$ and injected into LAD artery by retrograde perfusion through aorta, and the dye was circulated and uniformly distributed, except in the portion of the heart previously perfused by the occluded coronary artery. The heart was quickly excised and both atria and right ventricle were removed. The LV was weighed and sliced horizontally to 6 slices. After being weighted individually, the slices were incubated in $1 \%$ TTC prepared with $1 \times$ PBS for $8-15 \mathrm{~min}$ at $37{ }^{\circ} \mathrm{C}$, fixed in $10 \%$ neutral buffered formaldehyde for 24-48 $\mathrm{h}$, and then photographed under a microscope with a digital camera.

The areas stained with Evans blue (blue area, Normal Zone), TTC (red staining, RR), and TTCnegative area (white area, IS) was measured digitally using Image Pro-plus (Version 6.0). The myocardial
IS was measured and expressed as a percentage of IS over the total RR. Infarct, at-risk, and non-ischemic areas were identified based on tissue staining and measured IS by computerized videoplanimetry.

\section{SXJ-Medicated Plasma Preparation}

Since SXJ is compounded with a mixture of herbs that are neither water-soluble nor fat-soluble, a custom plasma containing SXJ for in vitro experiments was formulated and produced. Fifteen rats were divided into two groups including control (as baseline), SXJ group, in which the rats were gavaged with SXJ at a dose of $90 \mathrm{mg} / \mathrm{kg}$ for 15 days. The blood was draw $120 \mathrm{~min}$ after the last gavage of SXJ, and centrifuged at $2,500 \times \mathrm{g}$ for $15 \mathrm{~min}$ to acquire the medicated serum.

\section{H9C2 Cardiomyocyte Culture and Phase II I/R} Protocol in vitro

To simulate the myocardial $\mathrm{I} / \mathrm{R}$ injury in vitro, rat $\mathrm{H} 9 \mathrm{C} 2$ cardiomyocyte were cultured with preincubation of $5 \mathrm{mmol} / \mathrm{L} \mathrm{Na}_{2} \mathrm{~S}_{2} \mathrm{O}_{4}$ for $1.5 \mathrm{~h}$. Rat $\mathrm{H} 9 \mathrm{C} 2$ cardiomyocyte cell line was obtained from the American Type Culture Collection (ATCC, Manassas, VA, USA). The H9C2 cells were maintained in DMEM supplemented with $10 \%$ fetal calf serum at $37{ }^{\circ} \mathrm{C}$ in $\mathrm{CO}_{2}$ incubation. The medium was replaced every 2-3 days, and cells were sub-cultured or subjected to experimental procedures at $80 \%-90 \%$ confluence.

In the phase II protocol, to evaluate cardioprotective effects of the SXJ-medicated serum against I/R injury on H9C2 cells, five groups ( $n=3$, for each group) were studied: control group, I/R group, LY group, PD group, and SXJ group. As shown in Figure $1 \mathrm{~B}, \mathrm{I} / \mathrm{R}$ group was subjected to $5 \mathrm{mmol} / \mathrm{L}$ sodium hydrosulfite $\left(\mathrm{Na}_{2} \mathrm{~S}_{2} \mathrm{O}_{4}\right)$ for $1.5 \mathrm{~h}$ followed by 2-h reperfusion with normal DMEM medium. LY and PD groups served as negative drug controls with inhibitor pretreatments, $10 \mathrm{umol} / \mathrm{L}$ LY294002 and $10 \mu \mathrm{mol} / \mathrm{L}$ PD98059, respectively, for $2 \mathrm{~h}$. SXJ group was pretreated with the $10 \%(\mathrm{v} / \mathrm{v}) \mathrm{SXJ}$-medicated serum for $24 \mathrm{~h}$ followed by $\mathrm{I} / \mathrm{R}$ according to previous publication. ${ }^{(12)}$ Normal cultured $\mathrm{H} 9 \mathrm{C} 2$ cells without any treatment were used as control.

\section{Nuclear and Cytoplasmic Protein Extraction}

The method of nuclear and cytoplasmic protein extraction has been described in detail in previous publication. ${ }^{(13)}$ Briefly, cardiac tissues were placed in a microcentrifuge and rat $\mathrm{H} 9 \mathrm{C} 2$ cardiomyocytes were 


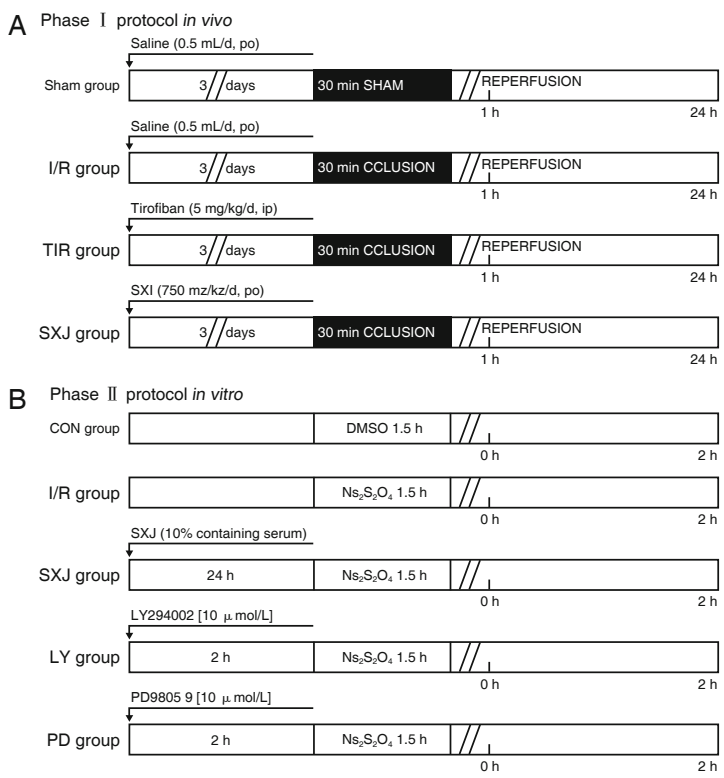

Figure 1. Phase I (A) and II (B) Experimental I/R Protocol

grown in 100-mm culture dishes. Nuclear proteins were isolated using the Fractionation kit according to the manufacturer's protocol (Thermo Fisher Scientific). Protein concentrations were determined using Bradford protein assay kit (Bio-Rad, USA). Cardiac tissues (50-100 mg) were cut into small pieces and placed in a microcentrifuge tube, washed with PBS, and centrifuged at $500 \times g$ for $5 \mathrm{~min}$. The supernatant was discarded to leave the cell pellet as dry as possible.

Tissue was homogenized with appropriate volume of CER I . Rat H9C2 cardiomyocytes were grown in 100-mm culture dishes, harvested with trypsin-EDTA and then centrifuged at $500 \times g$ for $5 \mathrm{~min}$. Cells were washed by suspending the cell pellet with PBS. Number of $10^{7}$ cells were transferred to a $1.5 \mathrm{~mL}$ microcentrifuge tube and centrifuged at $500 \times \mathrm{g}$ for $2-3 \mathrm{~min}$. The supernatant was discarded and the cell pellet was kept as dry as possible. Ice-cold CER I was added to the cell pellet. Subsequently, the tube was vortexed vigorously on the highest setting for $15 \mathrm{~s}$, and incubated on ice for $10 \mathrm{~min}$. Ice-cold CER II was added to the tube. The tube was vortexed for $5 \mathrm{~s}$, incubated on ice for $1 \mathrm{~min}$, and then centrifuged for $5 \mathrm{~min}$ at maximum speed in a microcentrifuge $(16,000 \times g)$. Immediately the supernatant (cytoplasmic extract) was transferred to a clean pre-chilled tube and placed on ice until use or storage. The insoluble (pellet) fraction produced in step 4, which contains nuclei, was suspended in ice-cold NER, and vortexed on the highest setting for $15 \mathrm{~s}$. Then the sample was placed on ice and continue vortexing for $15 \mathrm{~s}$ every $10 \mathrm{~min}$, for a total of $40 \mathrm{~min}$. Next the tube was centrifuged at maximum speed $(16,000 \times g)$ in a microcentrifuge for $10 \mathrm{~min}$, and then the supernatant (nuclear extract) fraction was transferred to a clean prechilled tube, and stored extracts at $-80^{\circ} \mathrm{C}$ until use.

\section{Western Blot Analysis}

The experiment of Western blot analysis of mice was harvested as listed as below. Mice were assigned to five groups ( $n=6$, for each group): sham group, $l / R$ group, LY group, PD group, and SXJ group. In the PD group, PD98059, an extracellular signal-regulated kinase pecific (ERK1/2) inhibitor, was administered $1 \mathrm{mg} / \mathrm{kg}$ via intragastric administration for 3 days. In the LY group, LY294002, a phosphatidylinositol-3-kinase (PI3-K) inhibitor, was intragastric administrated $7.5 \mathrm{mg} / \mathrm{kg}$ for 3 days, followed with $\mathrm{I} / \mathrm{R}$ surgery. Ischemia was elicited by a $30-\mathrm{min}$ coronary occlusion followed by $30-\mathrm{min}$ reperfusion, and then cardiac tissues were harvested and homogenized at $4{ }^{\circ} \mathrm{C}$ in RIPA lysis buffer. The cells were rinsed twice with PBS and homogenized in RIPA lysis buffer containing protease inhibitor PMSF. The insoluble protein lysate was removed by centrifugation at $12,000 \mathrm{r} / \mathrm{min}$ for $5 \mathrm{~min}$ at $4{ }^{\circ} \mathrm{C}$. Homogenized sample $(50 \mathrm{~g})$ of the protein lysate was resolved using 12\% SDS-polyacrylamide gel electrophoresis. The gels were transferred to polyvinylidene difluoride (PVDF) membranes by semidry electrophoretic transfer at $200 \mathrm{~mA}$ for $60 \mathrm{~min}$. The PVDF membranes were blocked $1 \mathrm{~h}$ in $5 \%$ milk at room temperature and subjected to Western blot analysis. Following antibodies were used in this study: anti-phospho-ERK1/2 (Thr202/Tyr204), anti-phospho-PKB (Ser473), anti-H3, and anti-PKB (Cell Signaling Technology, Beverly, MA, USA), anti-ERK1/2, anti-GSK-3B and anti-phospho (p)-GSK3b (Ser9), anti-GAPDH and anti-elF-5 (Santa Cruz Technology, Delaware, CA, USA).

\section{Statistical Analysis}

Data are reported as means \pm standard error of mean (SEM). Comparisons between groups were performed by ANOVA with the Newman-Keuls post hoc correction (GraphPad Prism version 4.0). Categorical data were analyzed by the chi-squared test. A $P$-value less than 0.05 was considered statistically significant.

\section{RESULTS}

SXJ Inhibited Myocardial I/R Injury in Mice As shown in Figure 2A, IS was clearly 
differentiated from RR and normal zone. As shown in Figure 2B, there were no significant differences in $\mathrm{RR}$ weight and in the ratio of RR/LV among the four groups $(P>0.05)$. Moreover, the ratio of IS/LV was also significantly higher in the $\mathrm{I} / \mathrm{R}$ group $(P<0.01)$ and lower in TIR and SXJ groups $(P<0.05$, Figure $2 \mathrm{C})$. At the end of $24 \mathrm{~h}$, the ratio of IS/RR was significantly higher in the I/R group than that of the sham group $(P<0.01)$, while it was lower in the TIR and SXJ groups than in the $1 / R$ group $(P<0.05$, Figure $2 D)$.
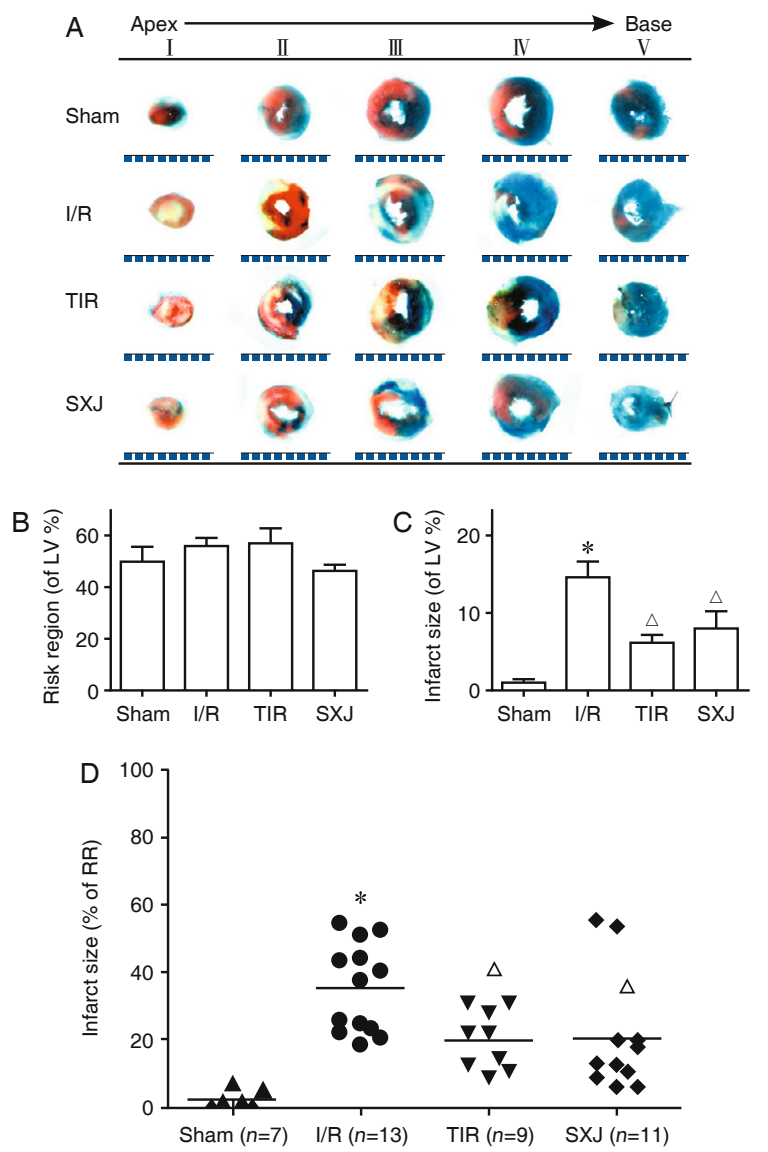

Figure 2. Dye Staining of the RRs and ISs of 4 Group Mice $(\bar{x} \pm$ SEM)

Notes: $(A)$ Photomicrographs $(\times 10)$ of heart sections obtained from mice subjected to myocardial I/R (30 $\mathrm{min} / 24 \mathrm{~h})$ treated with sham, I/R, TIR, and SXJ. Blue-stained portion: nonischemic, normal region; red-stained portion: I/R, risk but not infarcted region; unstained portion (white area): $/ / R$, infarcted region. Scale at bottom is in $\mathrm{mm}$. (B) Myocardial risk region (RR) expressed as percent of left ventricle (LV). Myocardial infarct size (IS) expressed as percent of LV (C) or RR (D). ${ }^{*} P<0.01$ vs. sham group, ${ }^{\triangle} P<0.05$ vs. I/R group.

Figure $3 \mathrm{~A}$ illustrated the representative electrocardiogram (ECG) complexes on the ischemia stage. Ischemia dramatically altered transmural ECG. The most notable features were a gradual increasing inversion of the T-wave (as the blcak arrow indicated). At the reperfusion stage, ventricular premature beats were observed to trigger ventricluar tachycardia for $5 \mathrm{~s}$ (Figure 3B) and then transfered to sinus rhythm (Figure 3C). Frequently, the atrial premature beats (Figure 3D) and ventricular premature beats ventricular contractions (Figure 3E) were occurred at the onset of reperfusion in the I/R model. The results (Figure $3 F$ ) showed that arrhythmias occured frequently in I/R group (incidence:71.4\% but significantly reduced in SXJ mice (incidence: $22.2 \%, P<0.05$ ).

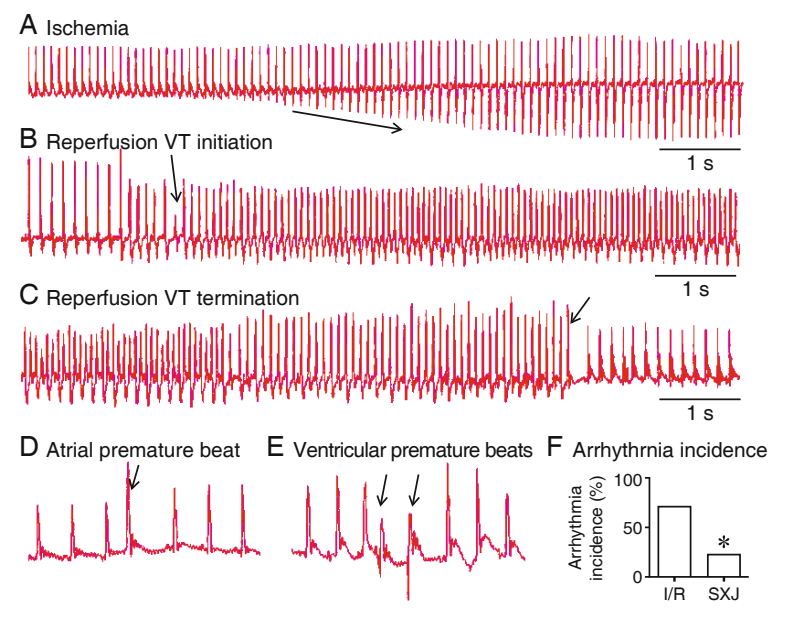

Figure 3. Reduced Incidence of Arrhythmia in SXJ Group

Notes: Representative ECG complexes in ischemia stage $(A)$, reperfusion VT initiation $(B)$, and reperfusion VT termination (C). Atrial premature beat (D) and ventricular premature beats (E) occurred frequently. Incidence of arrhythmia were reduced in SXJ group (F). ${ }^{*} P<0.05$ vs. I/R group

\section{SXJ Inhibited Myocardial Ischemia Injury in Rats}

As shown in Figure 4, cTnl, CK, AST, and $\alpha$-HBD were increased after ISO administration, but such increases were suppressed significantly by SXJ stimulation $(P<0.01)$. Furthermore, heart weight $(\mathrm{HW})$, the ration of heart weight to body weight (HW/BW), the ratio of heart weight to tibial length (HW/TL), and the ratio of lung weight to tibial length (Lung/TL) were all increased after ISO administration (Appendix 1). After SXJ stimulation, all these indices (HW, HW/BW, $\mathrm{HW} / \mathrm{TL}$, and Lung/TL) were decreased significantly $(P<0.01$, Appendix 1).

Phosphorylation of AKT/GSK3 $\beta$ and GATA4 Expression Were Enhanced in SXJ Group after I/R Surgery

As shown in Figure 5, phosphorylations of both ERK1/2 and AKT were enhanced after I/R surgery, and reduced after pre-incubation with specific ERK1/2 inhibitor (Figure 5A) and PI3K inhibitor $(P<0.05$, Figure 
$5 B)$, Interestingly, the phosphorylations of AKT, but not ERK1/2, was increased more in SXJ group than in the I/R group $(P<0.05)$.

Subsequently, the phosphorylation of GSK3 $\beta$, increased in response to I/R surgery and inhibited by PI3K inhibitor, LY294002, in consistence with the notion that AKT is one of the main RISK pathways in I/R injury. In addition, the phosphorylation of GSK3 $\beta$ was further increased after SXJ stimulation, compared with the I/R mice ( $P<0.05$, Figure $5 \mathrm{C})$. The expression of protein GATA4 in nuclear was enhanced in I/R group significantly (vs. sham, $P<0.01$ ), and inhibited in LY group. (vs. I/R, $P<0.01$ ). Compared with the I/R group, the GATA4 expression was further increased in SXJ group $(P<0.05$, Figure 5D).

\section{Enhanced AKT/GSK3 $\beta$ Phosphorylation and} GATA4 Expression by SXJ Followed by $\mathrm{Na}_{2} \mathrm{~S}_{2} \mathrm{O}_{4}$ Stimulation in H9C2 Cardiomyocytes

As shown in Figure 6, the phosphorylations of both ERK1/2 and AKT were enhanced after $\mathrm{Na}_{2} \mathrm{~S}_{2} \mathrm{O}_{4}$ stimulation but blocked by PD98059 (Figure 6A) and LY294002 ( $P<0.05$, Figures $6 B)$, which supported that the $\mathrm{Na}_{2} \mathrm{~S}_{2} \mathrm{O}_{4}$ simulated in vitro I/R model was successful. The phosphorylations of AKT was increased, while ERK1/2 phosphorylation were decreased in response to $10 \%$ SXJ-mediated plasma, compared with the $\mathrm{Na}_{2} \mathrm{~S}_{2} \mathrm{O}_{4}$ simulated I/R injury in vitro $(P<0.05)$. Next, the phosphorylation of GSK3 $\beta$ was increased in response to $\mathrm{Na}_{2} \mathrm{~S}_{2} \mathrm{O}_{4}$ stimulation and blocked by LY294002 (Figure 6C). Further, the phosphorylation of GSK3 $\beta$ was enhanced after preincubation with $10 \%$ SXJ-medicated plasma $(P<0.05)$. The nuclear GATA4 protein from rat I/R injury H9C2 cardiomyocytes were compared with the SXJ group, and it was found that the expression of protein GATA4 in nuclear was enhanced significantly in response to SXJ stimulation $(P<0.05$, Figure 6D).

Based on the results of the current study, a working model for SXJ's protective effects against I/R
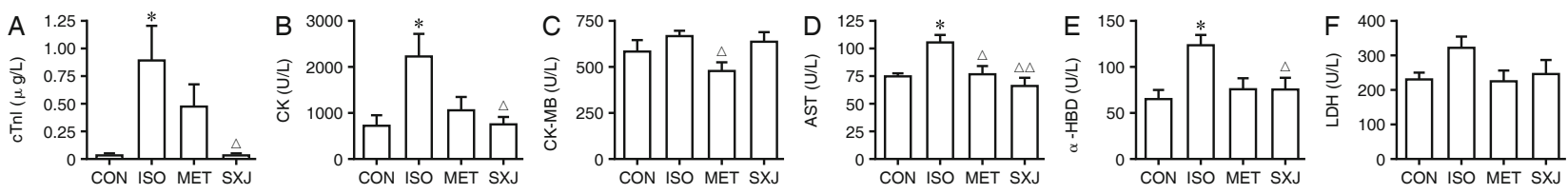

Figure 4. Effects of SXJ on Serum Index of Myocardial Injury Measured by ELISA in Rats $(\bar{x} \pm S E M, n=6-7)$ Notes: ${ }^{*} P<0.05$ vs. control group; ${ }^{\wedge} P<0.05,{ }^{\triangle} \mathrm{P}<0.01$ vs. ISO group; CON: control, ISO: isoproterenol, MET: metprolol.
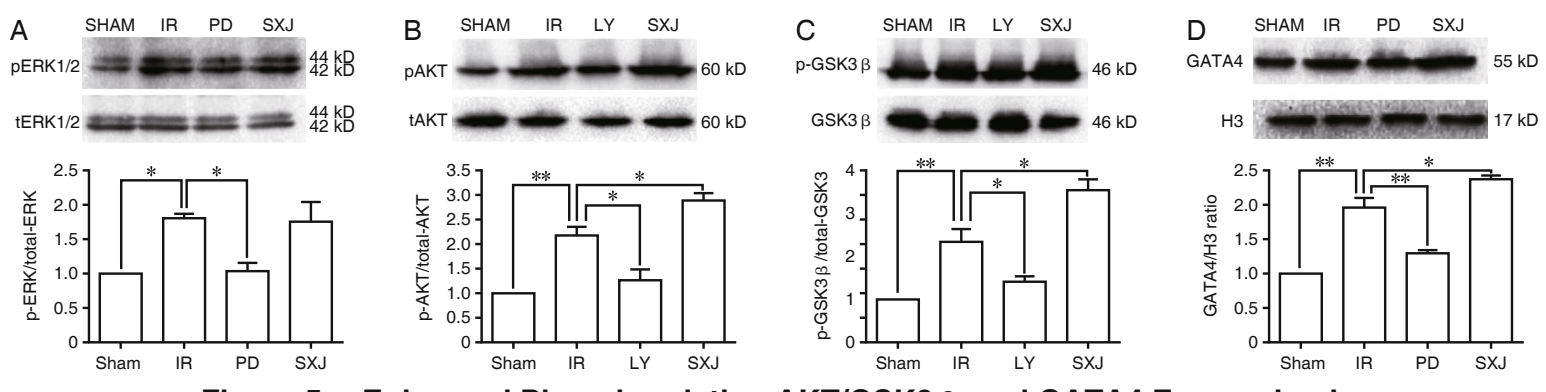

Figure 5. Enhanced Phosphorylation AKT/GSK3 $\beta$ and GATA4 Expression in SXJ group after I/R Surgery $(\bar{x} \pm S E M, n=3)$

Notes: Data were expressed as fold changes from relative total protein and GATA4/H3, ${ }^{*} P<0.05,{ }^{* *} P<0.01$.
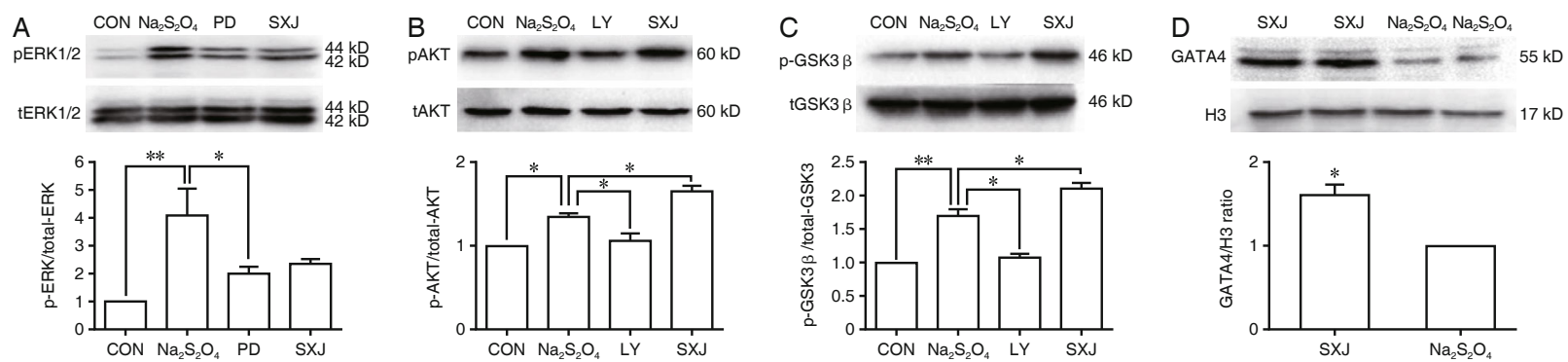

Figure 6. Enhanced AKT/GSK3 $\beta$ Phosphorylation and GATA4 Expression by

SXJ Followed by $\mathrm{Na}_{2} \mathrm{~S}_{2} \mathrm{O}_{4}$ Stimulation in $\mathrm{H} 9 \mathrm{C} 2$ Cardiomyocytes $(\overline{\mathrm{x}} \pm \mathrm{SEM}, \boldsymbol{n}=3)$

Notes: Data were expressed as fold changes from relative total protein and GATA4/H3, ${ }^{*} P<0.05,{ }^{* *} P<0.01$. 
injury was formulated (Figure 7). SXJ activates AKT/ GSK3 $\beta$ /GATA4 signaling pathways (one of the main RISK pathways), so as to protect against myocardial I/R injury. Whereas, ERK1/2, the other main RISK pathway, is not participated in the SXJ anti-myocardial I/R injury process.

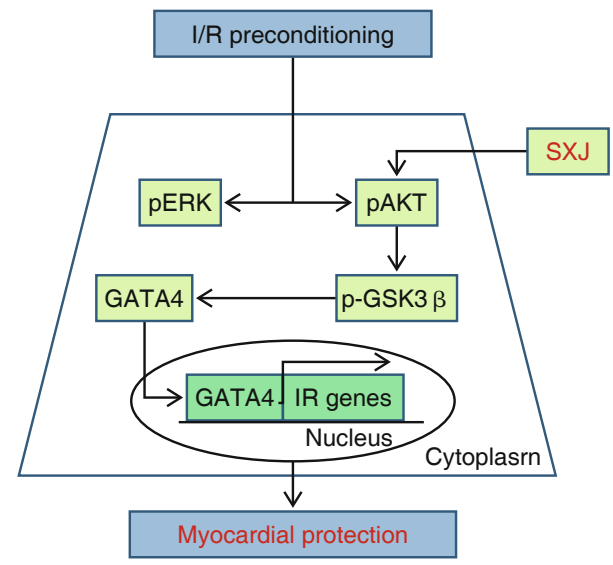

Figure 7. A Model of Pathways in the CardioProtection of SXJ in Response to Myocardial I/R Injury Notes: I/R preconditioning activates phosphorylation of ERK1/2, AKT and GSK3 $\beta$, enhances nucleus expression of transcript factor GATA4, thereby preventing myocardial I/R injury. SXJ may increase the phosphorylation of AKT/GSK3 $\beta$, enhancing the nucleus expression of GATA4, leading to inhibiting myocardial I/R injury. ERK1/2 pathway did not participate in SXJ anti-l/R injury process.

\section{DISCUSSION}

The present study was the mechanistic investigation of SXJ's protective effects against myocardial $\mathrm{I} / \mathrm{R}$ injury in mice. The results showed that SXJ-induced cardio-protection was mediated through an AKT/GSK3 $\beta$ /GATA4-dependent mechanism, which provided an experimental proof and support for the clinical application of SXJ in patients.

One of the classic mechanisms of myocardial I/R injury is RISK signaling pathway, which provides pharmacological targets to salvage viable myocardium and limit infarct size during myocardial I/R injury. We evaluated two key players in the RISK pathway, ERK $1 / 2$ and AKT proteins. ${ }^{(14,15)}$ Our results showed that SXJ treatment had little influence on ERK1.2 signaling pathway, while promoted the phosphorylation of AKT after I/R. These results suggested that AKT/ GSK3 $\beta$ mediated the SXJ-induced cardioprotection against acute myocardial I/R injury. The zinc-finger containing transcription factor GATA4 has been ascribed to many vital functions in the heart, ranging from early development of cardiomyocytes to adult cardiac hypertrophic response. ${ }^{(16)}$ Both ERK1/2 and AKT activities were necessary for increasing GATA4 DNA binding in stressed hearts. ${ }^{(17)}$ Overexpression of GATA4, a molecular marker of cardiac hypertrophy, by adenoviral gene transfer induced cardiomyocyte hypertrophy. ${ }^{(18)}$ However, the role of GATA4 in heart diseases is still unclear. Rysä, et al ${ }^{(19)}$ reported that GATA-4 could inhibit apoptosis and enhance angiogenesis in the infarcted myocardium. Inhibition of GATA-4 DNA-binding activity by a dominant negative mutant of GATA4 induced apoptosis. ${ }^{(20)}$ We found that GATA4 expression was enhanced after SXJ treatment in response to $\mathrm{I} / \mathrm{R}$ process (Figures 5 and 6), which revealed that GATA4 was participated in $\mathrm{I} / \mathrm{R}$ process and might be beneficial, in consistence with the antiapoptosis role of GATA4 on in AMI. Therefore, our findings contributed to understanding the molecular mechanisms of cardiac protection of SXJ. The functions of GATA4 in I/R injury are worth further studying.

Since there are multiple active ingredients in SXJ, one may postulate that SXJ could affect multiple mechanisms of myocardia I/R injury. However, our present study showed that SXJ inhibited I/R injury via a single signaling pathway, AKT/GSK3 $\beta$ /GATA4 pathway, and thus, provided an experimental support to the clinical effectiveness of SXJ treatment in improving cardiac function of patients with angina pectoris and AMI.

The mechanistic studies in the present study were limited by the in vivo and in vitro experimental models, and by using inhibitors, which might have limited specificity and efficiency in blocking the proteins. Further studies combining pharmacological and genetic techniques may be needed to investigate the signaling pathway of SXJ in the RISK signaling pathway. Due to the composition complexity of SXJ, using SXJ-mediated plasma produced a reasonable in vitro cultured cell model and as a platform to study SXJ. However, the SXJ-medicated plasma method could not differentiate the effects of components of SXJ in its anti-I/R injury effects. Further studies are needed to identify components of SXJ that plays anti-I/R injury role at molecular level.

In general, this study demonstrated that SXJ protected against myocardial I/R injury via activating AKT/GSK3 $\beta$ and GATA4 signaling pathways. The in vivo studies showed that SXJ reduced the size of 
infarction by $\mathrm{I} / \mathrm{R}$ injury, and thus, experimentally proved the effectiveness of SXJ as a clinical therapy to protect against ischemia reperfusion injury at an early stage. The SXJ-induced cardio-protection can help to explain the improved outcomes in patients with acute coronary syndrome after using SXJ and may lead to development of better therapies and applications of SXJ.

\section{Conflict of Interest}

The authors declare no competing financial interests.

\section{Author Contributions}

Zhang MZ and Qi JY conceived and designed the experiments, Tan YF and Yu J performed the experiments, Tan YF and $\mathrm{Yu} \mathrm{J}$ analyzed the data, Pan WJ contributed reagents/ materials/analysis tools. Zhang MZ and Qi JY wrote the manuscript. All authors read and approved the final version of the manuscript.

Electronic Supplementary Material: Supplementary materials (Appendixes) are available in the online version of this article at https://doi.org/10.1007/s11655-020-2726-2.

\section{REFERENCES}

1. Lakota J. Molecular mechanism of ischemia-reperfusion injury after myocardial infarction and its possible targeted treatment. Int J Cardiol 2016;220:571-572.

2. GuoY, Wu WJ, Qiu Y, Tang XL, Yang Z, Bolli R. Demonstration of an early and a late phase of ischemic preconditioning in mice. Am J Physiol 1998;275:H1375-87.

3. Lejay A, Fang F, John R, Van JA, Barr M, Thaveau F, et al. Ischemia reperfusion injury, ischemic conditioning and diabetes mellitus. J Mol Cell Cardiol 2016;91:11-22.

4. Chinese Pharmacopoeia Commission. Pharmacopoeia of the People's Republic of China. Beijing: Chinese Medical Science and Technology Press; 2015:1341.

5. Duan X, Zhou L, Wu T, Liu G, Qiao J, Wei J, et al. Chinese herbal medicine Suxiao Jiuxin Wan for angina pectoris. Cochrane Database Syst Rev 2008;23:CD004473.

6. Lu Z, Zhang Y, Zhuang P, Zhang J, Zhou H, Zhang M, et al. Protective effect of Suxiao Jiuxin Pill, a traditional Chinese medicine, against acute myocardial ischemia in dogs. BMC Complement Altern Med 2015;15:373-380.

7. Guo QX, Zhang J, Li YQ, Zhang GF. Study on antiatherosclerotic effect of Suxiao Jiuxin Pill and its mechanism. Afr J Tradit Complement Altern Med 2013;11:97-102.

8. Bai XY, Zhang P, Yang Q, Liu XC, Wang J, Tong YL, et al. Suxiao Jiuxin Pill induces potent relaxation and inhibition on contraction in human artery and the mechanism. Evid Based Complement Altern Med 2014;2014:956924.

9. Qi J, Yu J, Wang L, Guo L, Ma S, Huang D, et al. Tongguan
Capsule protects against myocardial ischemia and reperfusion injury in mice. Evid Based Complement Altern Med 2013;2013:159237.

10. Qi JY, Wang L, Gu DS, Guo LH, Zhu W, Zhang MZ. Protect effects of Danlou Tablet against murine myocardial ischemia and reperfusion injury in vivo. Chin $\mathrm{J}$ Integr Med 2018;24:613-620.

11. Qi JY, Yu J, Huang DH, Guo LH, Wang L, Huang X, et al. Salvianolate reduces murine myocardial ischemia and reperfusion injury via ERK1/2 signaling pathways in vivo. Chin J Integr Med 2017;23:40-47.

12. Jiang YR, Miao Y, Yang L, Xue M, Guo CY, Ma XJ, et al. Effect of Chinese herbal drug-containing serum for activating-blood and dispelling-toxin on ox-LDL-induced inflammatory factors' expression in endothelial cells. Chin J Integr Med 2012;18:30-33.

13. Liao WQ, Wang SY, Han CD, Zhang YY. 14-3-3 Proteins regulate glycogen synthase $3 \beta$ phosphorylation and inhibit cardiomyocyte hypertrophy. FEBS Journal 2005;272:1845-1854.

14. Das A, Xi L, Kukreja RC. Protein kinase G-dependent cardioprotective mechanism of phosphodiesterase- 5 inhibition involves phosphorylation of ERK and GSK3beta. J Biol Chem 2008;283:29572-29585.

15. Wang J, Ji SY, Liu SZ, Jing R, Lou WJ. Cardioprotective effect of breviscapine: inhibition of apoptosis in $\mathrm{H} 9 \mathrm{C} 2$ cardiomyocytes via the PI3K/AKT/eNOS pathway following simulated ischemia/ reperfusion injury. Pharmazie 2015;70:593-597.

16. Meganathan K, Sotiriadou I, Natarajan K, Hescheler J, Sachinidis A. Signaling molecules, transcription growth factors and other regulators revealed from in vivo and in vitro models for the regulation of cardiac development. Int $\mathrm{J}$ Cardiol 2015;183:117-128.

17. Xu X, Zhang L, Liang J. Rosuvastatin prevents pressure overload-induced myocardial hypertrophy via inactivation of the AKT, ERK1/2 and GATA4 signaling pathways in rats. Mol Med Rep 2013;8:385-392.

18. Liang Q, De Windt, LJ, Witt SA, Kimball TR, Markham BE, Molkentin JD. The transcription factors GATA4 and GATA6 regulate cardiomyocyte hypertrophy in vitro and in vivo. J Biol Chem 2001;276:30245-30253.

19. Rysä J, Tenhunen O, Serpi R, Soini $Y$, Nemer M, Leskinen $\mathrm{H}$, et al. GATA-4 is an angiogenic survival factor of the infarcted heart. Circ Heart Fail 2010;3:440-450.

20. Kim Y, Ma AG, Kitta K, Fitch SN, Ikeda T, Ihara $Y$, et al. Anthracycline-induced suppression of GATA-4 transcription factor: implication in the regulation of cardiac myocyte apoptosis. Mol Pharmacol 2003;63:368-377.

(Accepted June 13, 2018; First Online June 10, 2020) Edited by ZHANG Wen 